\title{
Introduction to the 37th International Conference on Logic Programming Special Issue I
}

\author{
ALEX BRIK \\ Google Inc., Mountain View, CA, USA \\ (e-mail: abrik@google.com) \\ ANDREA FORMISANO \\ Università di Udine, Udine, Italy \\ (e-mail: andrea.formisano@uniud.it) \\ YANHONG ANNIE LIU \\ Stony Brook University, Stony Brook, New York, USA \\ (e-mail: liu@cs.stonybrook.edu) \\ JOOST VENNEKENS \\ KU Leuven, De Nayer Campus Sint-Katelijne-Waver, Belgium \\ (e-mail: joost.vennekens@kuleuven.be) \\ submitted 08 September 2021; accepted 09 September 2021
}

This issue and its companion, the subsequent issue in this volume, contain selected papers of the 37th International Conference on Logic Programming (ICLP 2021), held online from September 20 to 27, 2021. The Association of Logic Programming (ALP) Executive Committee decided to hold ICLP 2021 as a fully virtual event because of the continuing coronavirus (COVID-19) pandemic.

Since the first conference held in Marseille in 1982, ICLP has been the premier international event for presenting research in logic programming. Contributions to ICLP 2021 were sought in all areas of logic programming, including but not restricted to:

Foundations: Semantics, Formalisms, Nonmonotonic reasoning, Knowledge representation.

Languages issues: Concurrency, Objects, Coordination, Mobility, Higher order, Types, Modes, Assertions, Modules, Meta-programming, Logic-based domainspecific languages, Programming techniques.

Programming support: Program analysis, Transformation, Validation, Verification, Debugging, Profiling, Testing, Execution visualization.

Implementation: Compilation, Virtual machines, Memory management, Parallel and Distributed execution, Constraint handling rules, Tabling, Foreign interfaces, User interfaces.

Related Paradigms and Synergies: Inductive and coinductive logic programming, Constraint logic programming, Answer set programming, Interaction with SAT, SMT and CSP solvers, Theorem proving, Argumentation, Probabilistic programming, Machine learning. 
Applications: Databases, Big data, Data integration and federation, Software engineering, Natural language processing, Web and semantic web, Agents, Artificial intelligence, Computational life sciences, Cyber-security, Robotics, Education.

Besides the main track, ICLP 2021 hosted the following two tracks. These tracks had their own track chairs, submission requirements, and evaluation criteria.

Applications Track: This track invited submissions of papers on emerging and deployed applications of LP, describing all aspects of the development, deployment, and evaluation of logic programming systems for solving real-world problems, including interesting case studies and benchmarks, and discussing lessons learned.

Recently Published Research Track: This track provided a forum for discussing important results in the broad area of logic programming that appeared, from January 2019 onwards, in selective journals and conferences but have not been presented at previous editions of ICLP.

ICLP 2021 also hosted the following events as well as five additional workshops focused on special technical topics.

MentorLP - Mentoring Workshop on Logic Programming: The purpose of MentorLP was to support students and newcomers to pursue careers in logic programming research. This workshop held technical talks on cutting-edge research in logic programming, and mentoring sessions on how to prepare and succeed for a research career. MentorLP emphasized fostering and supporting diversity, equity, and inclusion.

Autumn School on Logic and Constraint Programming: The Autumn School was designed for those interested in learning advanced topics in logic programming and constraint programming. It consisted of four half-day tutorials.

Doctoral Consortium on Logic Programming: The Doctoral Consortium provided students with the opportunity to present and discuss their research directions, and to obtain feedback from both peers and experts in the field.

Logic and Constraint Programming Contest: The contest combined some features of Prolog programming competition, Answer Set Programming competition, and Constraint Programming competition. As in the previous edition, participants were not limited to use a single system and could also combine declarative and imperative programming languages.

The technical program of ICLP 2021 also included five invited talks by prominent researchers from academia and industry:

- Answering Questions using Neural Knowledge Representations William W. Cohen, Google AI

- Logic-Based Formulation of Ethical Principles John Hooker, Carnegie Mellon University

- Structural Characterizations of Rule-Based Languages Phokion Kolaitis, University of California, Santa Cruz, and IBM Research

- Combining Probability and First-Order Logic

Stuart Russell, University of California, Berkeley

- Why Datalog?

Jeffrey Ullman, Stanford University

and two invited panels: 
Datalog Perspectives: This panel was chaired by David S. Warren (Stony Brook University and XSB, Inc.). The panelists were David Maier (Portland State University), Moshe Vardi (Rice University), and Jeffrey Ullman (Stanford University). The panel was originally planned to be immediately after Ullman's invited talk, but Ullman shared his invited talk time with Maier and Vardi before the panel discussions. Maier's talk was titled The Downs and Ups of Datalog, and Vardi's talk was titled Datalog + + and Datalog-.

No Logic is an Island: Internal and External Integration of Logic Programming Paradigms: This panel was chaired by Joost Vennekens (KU Leuven). The panelists were Marc Denecker (KU Leuven), Manuel Hermenegildo (IMDEA and Universidad Politécnica de Madrid), Francesco Ricca (University of Calabria), Theresa Swift (Universidade Nova de Lisboa), and Jan Wielemaker (VU University of Amsterdam).

Furthermore, after a thorough examination of citation indices (e.g., Google Scholar, Web of Science, Scopus) for follow-up works, two test-of-time awards were identified:

- John Alan Robinson 20-Year Test-of-Time Award: Marc Denecker, Nikolay Pelov, and Maurice Bruynooghe. Ultimate Well-Founded and Stable Semantics for Logic Programs with Aggregates, in Proceedings of ICLP 2001.

- Alain Colmerauer 10-Year Test-of-Time Award: Fabrizio Riguzzi and Terrance Swift. The PITA system: Tabling and Answer Subsumption for Reasoning Under Uncertainty, in Proceedings of ICLP 2010.

The organizers of ICLP 2021 were:

General Chair

Ricardo Rocha, University of Porto, Portugal

\section{Program Chairs}

Andrea Formisano, University of Udine, Italy

Y. Annie Liu, Stony Brook University, USA

Organizing and Publicity Chair

Miguel Areias, University of Porto, Portugal

Workshops Chair

Nicos Angelopoulos, Cardiff University, UK

Autumn School and Doctoral Consortium Chairs

Bart Bogaerts, Vrije Universiteit Brussel, Belgium

Carmine Dodaro, University of Calabria, Italy

Programming Contest Chair

Mario Alviano, University of Calabria, Italy

Applications Track Chairs

Alex Brik, Google Inc., USA

Joost Vennekens, KU Leuven, Belgium

Recently Published Research Track Chairs

Gian Luca Pozzato, University of Turin, Italy

Neng-Fa Zhou, CUNY Brooklyn College and Graduate Center, USA

MentorLP - Mentoring Workshop on Logic Programming Chairs

Veronica Dahl, Simon Fraser University, Canada

Paul Fodor, Stony Brook University, USA 
ICLP 2021 implemented the hybrid publication model - with both Theory and Practice of Logic Programming (TPLP) journal articles and Technical Communication (TC) papers - as decided in 2010 by ALP. Papers judged by the Program Committee to be of the highest quality were selected for rapid publications in a special issue of TPLP. The TCs include papers judged by the Program Committee to be of good quality but not yet of the standard required to be published in TPLP, or extended abstracts abridged from such papers if the authors chose. The TCs also include accepted short papers, accepted extended abstracts from the Recently Published Research Track, abstracts of invited talks and panels, and dissertation project descriptions from the Doctoral Consortium.

We received 74 submissions of abstracts, of which 68 resulted in completed submissions, distributed as follows: for ICLP Main Track, 43 full papers and 5 short papers, for Applications Track, 13 full papers and 2 short papers, and for Recently Published Research Track, 5 extended abstracts. The Program Chairs organized the reviewing process by the Program Committee with support of several external reviewers. Each technical paper was reviewed by at least three reviewers, who provided detailed written evaluations. This enabled a subset of the papers to be short-listed as candidates for rapid publication in TPLP. The authors of these papers revised their submissions in light of the reviewers' comments and suggestions, and all these papers were subject to a second round of reviewing, and a few of them an additional round of reviewing. Of these candidate papers, 20 were accepted for publication in TPLP. In addition, the Program Committee recommended 23 papers to be accepted as Technical Communications, to appear in Electronic Proceedings in Theoretical Computer Science (EPTCS), either as full papers or as extended abstracts (one of these papers was withdrawn).

The 20 papers accepted for publication in TPLP appear in two issues, the present issue and the subsequent one, each containing 10 papers. The present issue contains the following papers.

\section{Papers from the Main Track:}

- Mario Alviano, Sotiris Batsakis, George Baryannis. Modal Logic S5 Satisfiability in Answer Set Programming.

- Damiano Azzolini, Fabrizio Riguzzi. Optimizing Probabilities in Probabilistic Logic Programs.

- Elena Bellodi, Marco Gavanelli, Riccardo Zese, Evelina Lamma, Fabrizio Riguzzi. Non-ground Abductive Logic Programming with Probabilistic Integrity Constraints.

- Viktor Besin, Markus Hecher, Stefan Woltran. Utilizing Treewidth for Quantitative Reasoning on Epistemic Logic Programs.

- Loris Bozzato, Thomas Eiter, Rafael Kiesel. Reasoning on Multi-Relational Contextual Hierarchies via Answer Set Programming with Algebraic Measures.

- Francesco Calimeri, Marco Manna, Elena Mastria, Maria Concetta Morelli, Simona Perri, Jessica Zangari. I-DLV-sr: A Stream Reasoning System based on IDLV.

- Angelos Charalambidis, Panos Rondogiannis, Antonis Troumpoukis. A Logical Characterization of the Preferred Models of Logic Programs with Ordered Disjunction.

- Wolfgang Faber, Michael Morak, Lukáš Chrpa. Determining Action Reversibility in STRIPS Using Answer Set and Epistemic Logic Programming. 
- Jorge Fandinno, François Laferrière, Javier Romero, Torsten Schaub, Tran Cao Son. Planning with Incomplete Information in Quantified Answer Set Programming.

- Pascual Julián-Iranzo, Fernando Sáenz-Pérez. Planning for an Efficient Implementation of Hypothetical Bousi Prolog.

The subsequent issue contains the following papers.

Papers from the Main Track:

- Bishoksan Kafle, Graeme Gange, Peter J. Stuckey, Peter Schachte, Harald Sondergaard. Transformation-Enabled Precondition Inference.

- Tuomo Lehtonen, Johannes P. Wallner, Matti Järvisalo. Harnessing Incremental Answer Set Solving for Reasoning in Assumption-Based Argumentation.

- Vladimir Lifschitz. Here and There with Arithmetic.

- Jukka Pajunen, Tomi Janhunen. Solution Enumeration by Optimality in Answer Set Programming.

- Miguel A. Sanchez-Ordaz, Isabel Garcia-Contreras, Victor Perez-Carrasco, Jose F. Morales, Pedro lopez-Garcia, Manuel V. Hermenegildo. VeriFly: On-the-fly Assertion Checking via Incrementality.

- Kylian Van Dessel, Jo Devriendt, Joost Vennekens. FOLASP: FO(.) as Input Language for Answer Set Solvers.

- Felix Weitkämper. An Asymptotic Analysis of Probabilistic Logic Programming, with Implications for Expressing Projective Families of Distributions.

Papers from the Applications Track:

- Paul S. Brown, Vania Dimitrova, Glen Hart, Anthony G. Cohn, Paulo Moura. Refactoring the Whitby Intelligent Tutoring System for Clean Architecture.

- Carmine Dodaro, Giuseppe Galatà, Andrea Grioni, Marco Maratea, Marco Mochi, Ivan Porro. An ASP-based Solution to the Chemotherapy Treatment Scheduling Problem.

- Müge Fidan, Esra Erdem. Knowledge-Based Stable Roommates Problem: A RealWorld Application.

We are deeply indebted to the Program Committee members and external reviewers. The conference would not have been possible without their enthusiastic and outstanding work.

The Program Committee members of ICLP 2021 were:

$\begin{array}{lll}\text { Salvador Abreu } & \text { Giovambattista Ianni } & \text { Orkunt Sabuncu } \\ \text { Mario Alviano } & \text { Katsumi Inoue } & \text { Chiaki Sakama } \\ \text { Marcello Balduccini } & \text { Tomi Janhunen } & \text { Vitor Santos Costa } \\ \text { Roman Barták } & \text { Michael Kifer } & \text { Torsten Schaub } \\ \text { Pedro Cabalar } & \text { Ekaterina Komendantskaya } & \text { Konstantin Schekotihin } \\ \text { Manuel Carro } & \text { Nicola Leone } & \text { Tom Schrijvers } \\ \text { Stefania Costantini } & \text { Michael Leuschel } & \text { Tran Cao Son } \\ \text { Marina De Vos } & \text { Ondřej Lhoták } & \text { Theresa Swift } \\ \text { Agostino Dovier } & \text { Vladimir Lifschitz } & \text { Paul Tarau } \\ \text { Inês Dutra } & \text { Fangzhen Lin } & \text { Tuncay Tekle } \\ \text { Thomas Eiter } & \text { Francesca Alessandra Lisi } & \text { Michael Thielscher }\end{array}$




$\begin{array}{lll}\text { Esra Erdem } & \text { Jorge Lobo } & \text { Mirek Truszczyński } \\ \text { Wolfgang Faber } & \text { Viviana Mascardi } & \text { Allen van Gelder } \\ \text { Sarah Alice Gaggl } & \text { Thomas Meyer } & \text { German Vidal } \\ \text { Marco Gavanelli } & \text { Jose F. Morales } & \text { Alicia Villanueva } \\ \text { Martin Gebser } & \text { Manuel Ojeda-Aciego } & \text { Toby Walsh } \\ \text { Michael Gelfond } & \text { Carlos Olarte } & \text { Antonius Weinzierl } \\ \text { Laura Giordano } & \text { Magdalena Ortiz } & \text { Jan Wielemaker } \\ \text { Gopal Gupta } & \text { Mauricio Osorio } & \text { Stefan Woltran } \\ \text { Michael Hanus } & \text { Enrico Pontelli } & \text { Roland Yap } \\ \text { Manuel Hermenegildo } & \text { Francesco Ricca } & \text { Jia-Huai You }\end{array}$

The Program Committee members of the ICLP 2021 Applications Track were:

$\begin{array}{lll}\text { Mutsunori Banbara } & \text { Matti Järvisalo } & \text { Daniele Theseider Dupré } \\ \text { François Bry } & \text { Nikos Katzouris } & \text { Paolo Torroni } \\ \text { Francesco Calimeri } & \text { Zeynep Kiziltan } & \text { Kewen Wang } \\ \text { Angelos Charalambidis } & \text { Marco Maratea } & \text { David Warren } \\ \text { Ferdinando Fioretto } & \text { Yunsong Meng } & \text { Fangkai Yang } \\ \text { Gerhard Friedrich } & \text { Alessandra Mileo } & \text { Zhizheng Zhang } \\ \text { Jianmin Ji } & \text { Mohan Sridharan } & \end{array}$

The external reviewers were:

$\begin{array}{lll}\text { Sotiris Batsakis } & \text { Spencer Killen } & \text { Etienne Tignon } \\ \text { Michael Bernreiter } & \text { Bruno Pereira } & \text { Huaduo Wang } \\ \text { Francois Bry-Hausser } & \text { Jacques Robin } & \text { Philipp Wanko } \\ \text { Jose-Luis Carballido } & \text { Elmer Salazar } & \text { Jessica Zangari } \\ \text { Carmine Dodaro } & \text { Zeynep G. Saribatur } & \text { Claudia Zepeda Cortes } \\ \text { Biqing Fang } & \text { Mantas Simkus } & \end{array}$

We would also like to express our gratitude to all other organizers of ICLP 2021. Our gratitude must be extended to Thomas Eiter, President of ALP; Marco Gavanelli, ALP Conference Coordinator; and members of ALP Board. Special thanks go to Mirek Truszczyński, Editor-in-Chief of TPLP, and the staff of Cambridge University Press for their assistance. Last but not least, we thank each author of every submitted paper, because their efforts keep the conference series move forward, and we thank all participants of ICLP 2021 for bringing and sharing their ideas and latest developments. 